\title{
Impact of maternal education level on live birth rate after in vitro fertilization: a retrospective cohort study
}

\author{
Xitong $\mathrm{Liu}^{1}$, Xin $\mathrm{Mu}^{1}$, Juanzi $\mathrm{Shi}^{1}$, and Haiyan Bai ${ }^{1}$ \\ ${ }^{1}$ Affiliation not available
}

May 18, 2021

\begin{abstract}
Objective To examine the association between maternal education level and live birth after in vitro fertilization (IVF). Design Retrospective cohort study. Setting Public fertility center in China. Population 41546 women who underwent the first cycle of fresh or frozen-thawed embryo transfer between 2014 and 2019. Methods The women were divided into four educational categories according to the level of education received (elementary school graduate or less, middle school graduate, high school graduate, college graduate or higher). Main outcome measures Live birth rates. Results Patients were grouped by maternal educational level: elementary school graduate or less $(n=1590)$, middle school graduate $(n=10996)$, high school graduate $(\mathrm{n}=8354)$, and college graduate or higher $(\mathrm{n}=20606)$. The live birth rate, miscarriage rate, and clinical pregnancy rate in elementary school graduate or less were lower compared to other groups. In multivariate logistic regression analysis, we fail to demonstrate a statistically significant relationship between educational level and live birth in middle school graduate (adjusted odds ratio [AOR] 0.96; 95\% confidence interval [CI], 0.84-1.09), high school graduate (AOR 1.01; 95\% CI, 0.87-1.14) or college graduate or higher (AOR 1.01; 95\% CI, 0.88-1.14) patients compared with the elementary school graduate or less reference group after adjusting for female age, infertility duration, BMI, EM thickness, no. of oocyte retrieved, infertility type, protocol in the fresh cycle, fertilization type, time of transfer and no. of embryo transferred. Conclusion No statistically significant relationship was identified between educational level and live birth in patients undergoing fresh or frozen embryo transfer.
\end{abstract}

Running title: Education level does not impact live birth

Impact of maternal education level on live birth rate after in vitro fertilization: a retrospective cohort study

Xitong Liu, ${ }^{\text {a }}$ Xin $\mathrm{Mu},{ }^{\mathrm{a}}$ Juanzi Shi, ${ }^{\text {a }}$ Haiyan Bai, ${ }^{\mathrm{a}}$

a The Assisted Reproduction Center, Northwest Women's and Children's Hospital, Xi'an, China

Correspondence: Haiyan Bai, The Assisted Reproduction Center, Northwest women's and Children's Hospital, Xi'an, China.

Email: flyindance29578@qq.com

ObjectiveTo examine the association between maternal education level and live birth after in vitro fertilization (IVF).

Design Retrospective cohort study.

Setting Public fertility center in China.

Population 41546 women who underwent the first cycle of fresh or frozen-thawed embryo transfer between 2014 and 2019. 
Methods The women were divided into four educational categories according to the level of education received (elementary school graduate or less, middle school graduate, high school graduate, college graduate or higher).

Main outcome measures Live birth rates.

Results Patients were grouped by maternal educational level: elementary school graduate or less ( $\mathrm{n}=1590)$, middle school graduate $(n=10996)$, high school graduate $(n=8354)$, and college graduate or higher $(n=20606)$. The live birth rate, miscarriage rate, and clinical pregnancy rate in elementary school graduate or less were lower compared to other groups. In multivariate logistic regression analysis, we fail to demonstrate a statistically significant relationship between educational level and live birth in middle school graduate (adjusted odds ratio $[\mathrm{AOR}]$ 0.96; 95\% confidence interval [CI], 0.84-1.09), high school graduate (AOR 1.01; 95\% CI, 0.87-1.14) or college graduate or higher (AOR 1.01; 95\% CI, 0.88-1.14) patients compared with the elementary school graduate or less reference group after adjusting for female age, infertility duration, BMI, EM thickness, no. of oocyte retrieved, infertility type, protocol in the fresh cycle, fertilization type, time of transfer and no. of embryo transferred.

Conclusion No statistically significant relationship was identified between educational level and live birth in patients undergoing fresh or frozen embryo transfer.

Keywords educational level, IVF, live birth rate

Tweetable abstract Maternal educational level does not influence live birth in IVF.

\section{Introduction}

Educational level has been shown to be an independent factor in access to health care [1]. According to the World Health Organization (WHO), lack of education is one of the contributing factors to increased maternal and child mortality and morbidity in developing countries [2]. In general, education has been shown to have a positive impact on different health issues.

Education influences lifestyles and social attitudes. Women with a higher education level are more likely to postpone marriage and childbearing. They comprise a large proportion of the IVF patient population. In addition, the expansion of higher educational attainment in women is a trend for the future. Women with higher education levels are more likely to correctly perform IVF protocol, thus may improve their clinical outcomes. Several studies have suggested the association between healthier lifestyle behaviors and higher education attainment [3-5]. However, the role of education in predicting varied clinical outcomes after IVF has rarely been studied. Thus, a better understanding of the association between education attainment and IVF outcomes would have broad social importance.

The aim of this study was to determine whether maternal education level is associated with live birth after IVF. Assessing the impact of maternal education level on reproduction outcomes may provide opportunities to identify deficiencies in maternal knowledge and modify them through counseling.

\section{Methods}

\section{Study design and patients}

This retrospective cohort study includes 41546 women who underwent fresh or frozen-thawed embryo transfer between January 2014 and December 2019 in the Center for Assisted Reproductive Technology of Northwest Women's and Children's Hospital, China. All the patients were followed up for one year after embryo transfer. The study protocol was approved by the ethics committee of Northwest Women's and Children's Hospital (No. 2019013). Because of the retrospective character of the study, informed consent was waived. Patient data were anonymized. Women who underwent the first cycle of fresh or frozen-thawed embryo transfer (FET) were eligible to participate. We excluded women with no embryo transferred. The women were divided into four educational categories according to the level of education received (elementary school graduate or less, middle school graduate, high school graduate, college graduate or higher). 


\section{IVF protocol}

Doctors chose different IVF protocols based on the patient's age and ovarian reserve. The GnRH agonist and GnRH antagonist protocols have been the main protocol in our center. Controlled ovarian hyperstimulation, oocyte retrieval, embryo transfer, and endometrial preparation for frozen-thawed embryo transfer (FET) were performed as previously described [6,7]. Briefly, for the GnRH agonist, patients used down-regulation from the mid-luteal phase of the previous cycle, recombinant FSH was started at 150-225IU/day when the pituitary had reached desensitization. For the GnRH antagonist protocol, recombinant FSH was initiated on day 2 of the cycle. GnRH antagonist was started when the dominant follicle reached $12-14 \mathrm{~mm}$ at $250 \mathrm{mg} / \mathrm{d}$. When two or more follicles reached $18 \mathrm{~mm}$, hCG was administrated at a dose of 4,000 to 10,000. Oocyte retrieval was carried out 34-36 h following hCG trigger. Conventional IVF or intracytoplasmic sperm injection (ICSI) was performed according to the male partner's semen quality.

\section{Outcome variables assessed}

Live birth was defined as the delivery of at least one live-born baby at greater than 24 weeks of gestational age. Clinical pregnancy was defined as the presence of at least one viable fetal cardiac activity at the 6-8 weeks transvaginal ultrasound. Miscarriage was defined as a spontaneous loss of a clinical pregnancy before 24 completed weeks of gestational age, in which the embryo(s) or fetus(es) is/are nonviable and is/are not spontaneously absorbed or expelled from the uterus. Preterm birth was defined as a birth that takes place after 24 weeks and before 37 completed weeks of gestational age.

\section{Statistical analysis}

Categorical variables were expressed as the number of cases (n) and percentage of occurrence (\%). The numerical data are presented as the mean \pm SEM and differences in parameters between the groups were compared using independent sample t-tests or $\chi 2$ tests as appropriate. Univariate and multivariate regression analyses were performed to determine the association between education level and live birth, excluding the interference of confounding factors. If adding a covariate to the model changed the estimated effect of a woman's educational level on live birth by $10 \%$ or more, the covariate was considered a confounder of the relationship between the woman's educational level and live birth and was included in the final multivariable models. We calculated crude odds ratios (OR) and adjusted ORs (aOR) with a 95\% confidence interval (CI). Interaction and stratified analyses were conducted according to confounders. Data were analyzed with the use of the statistical packages R (The R Foundation; http://www.r-project.org;version 3.4.3) and Empower (R) (www.empowerstates.com, $\mathrm{X} \& \mathrm{Y}$ solutions, inc. Boston, Massachusetts). The level of significance was set at $p<0.05$.

\section{Results}

Table 1 shows the baseline characteristics. We identified 41,546 cycles who met the study criteria, including 1590 women with elementary school graduate or less, 10996 women with middle graduate, 8354 with high school graduate, and 20606 with college graduate or higher. The distribution of baseline characteristics of the study population is presented in Table 1 . There was a significant difference in baseline characteristics between groups, with elementary school or less education level women more likely to be older, have longer infertility duration, higher BMI, more secondary infertility, IVF insemination, fresh embryo transfer, and double embryo transfer, while less no. of oocyte retrieved and agonist protocol in the fresh cycle. The live birth rate, miscarriage rate, and clinical pregnancy rate in elementary school graduate or less were lower compared to other groups.

Univariate analysis was performed to evaluate each variable's effect on the live birth rate (Table 2). Female age, infertility duration, BMI, secondary infertility, antagonist, and other protocol in the fresh cycle and frozen embryo transfer were negatively associated with live birth, while endometrial thickness, no. of oocyte retrieved, and double embryo transfer were positively associated with live birth. Interaction and stratified analyses were shown in Table S1. There were no significant interactions in any of the subgroups $(\mathrm{p}>0.05$ for all comparisons). 
Compared with women with an education corresponding to elementary school graduate or less, there was an increased rate of live birth among women with higher educational levels in the crude model. However, after adjusting for female age, infertility duration, BMI, EM thickness, no. of oocyte retrieved, infertility type, protocol in the fresh cycle, fertilization type, time of transfer, and no. of embryo transferred, women's educational level was not associated with live birth.

\section{Discussion}

\section{Main findings}

In this retrospective cohort study of 41546 women undergoing IVF with embryo transfer, we showed that live birth was not significantly different in women with different educational levels.

Higher educational level has been found to be related to lower incidence and prevalence of cardiovascular diseases, diabetes, cancers, and chronic respiratory diseases [8]. The higher educational level also leads to better use of health care services [9], more disease prevention in terms of screening for sexually transmitted disease [10], and a lower risk of low 5-min Apgar score [11]. However, few studies have focused on the relationship between maternal educational level and live birth after IVF.

In general, women with higher education are more likely to be involved in healthy behavior such as regular exercise, a healthy diet, and regular check-up. Maternal educational level has been shown to be a strong predictor of seeking medical help with infertility [12]. As shown in this study, women with higher educational levels were more likely to be thinner. At the same time, our findings showed that women with less education were older, have longer infertility duration.

Education is a vital determinant of fertility behavior and outcomes. The IVF treatment process is extremely complicated and requires efficient coordination of multiple links [13]. Women with higher educational levels have a higher chance of avoiding mistakes in the IVF process. There have been several studies that have examined the association between maternal educational level and fertility. Previous data from the demographic and health surveys confirms that higher education is associated with lower fertility [14]. The reason for reduced fertility is usually owned to birth postponement and the high opportunity cost of childbearing. More-educated women tend to marry later, have a week orientation towards having families [15]. However, poor fertility knowledge is a contributory factor to many couples not achieving their goal of parenthood [16]. Less-educated women may be left ill-equipped to make informed choices about their reproductive lives and relationships [17]. In addition, women with higher levels of education have lower rates of depressive symptoms in IVF treatment [18]. This may offset the detrimental effect of postponing parenthood.

\section{Strengths and limitations}

Strengths of our study include its large sample size, incorporation of women of all age ranges, and representation of patients from the full spectrum of IVF protocols. Another advantage of this study was that it included only single-center cycles, which minimized potential bias (e.g., laboratory conditions and the protocol of IVF). In addition, we adjusted more variables to make the result more reliable.

There are some limitations to this study, such as its retrospective nature, which reduces its direct application to clinical practice. Interpretation of the results must take into account the fact that the study was restricted to patients who underwent IVF with at least a basic level of income and equal access to health facilities.

\section{Interpretation}

The association between live birth after IVF and maternal educational level has not been well investigated previously. It remains unclear, whether women's educational level itself influences the clinical outcomes of IVF. One cohort study of 2569 women indicated that a woman's educational attainment was not associated with the likelihood of implantation failure, spontaneous abortion, or live birth [19], which is in accordance with our study. However, the women in this study were only divided into three educational categories: no college degree, college degree, and graduate attendance. The classification is fuzzy and does not apply to the current educational system. 


\section{Conclusion}

Our results demonstrated no relationship between maternal educational level and live birth in women undergoing IVF. While the findings of this study are valuable in contributing towards the current understanding of the relationship between educational attainment and pregnancy outcome, further research needs to be undertaken before the results can be extrapolated to the general population.

Disclosure of interests

None declared.

Contribution to authorship

XL designed the study. XM and JS conducted data collection, analysis and writing the manuscript. HB conceived and designed the study.

\section{Details of ethics approval}

This study was approved by the ethics committee of the hospital (number 2019013).

Funding

Supported by National Natural Science Foundation of China (No. 81771657 http://www.nsfc.gov.cn/) and General Projects of Social Development in Shaanxi Province(No. 2018SF-260).

\section{Acknowledgements}

The authors thank the staff from Northwest Women's and Children's Hospital for their assistance with the data collection.

\section{References}

1. Gilbert A, Benjamin A, Abenhaim HA. Does education level influence the decision to undergo elective repeat caesarean section among women with a previous caesarean section? Journal of obstetrics and gynaecology Canada 2010; 32: 942-7.

2. Princewill CW, De Clercq E, Riecher-Rössler A, Jegede AS, Wangmo T, Elger BS. Education and Reproductive Autonomy: The Case of Married Nigerian Women. Narrative inquiry in bioethics 2017; 7: 231-44.

3. Di Cesare M, Khang YH, Asaria P, Blakely T, Cowan MJ, Farzadfar F et al. Inequalities in noncommunicable diseases and effective responses. Lancet (London, England) 2013; 381: 585-97.

4. Townsend J, Roderick P, Cooper J. Cigarette smoking by socioeconomic group, sex, and age: effects of price, income, and health publicity. BMJ (Clinical research ed) 1994; 309: 923-7.

5. Leclerc A, Pietri F, Boitel L, Chastang JF, Carval P, Blondet M. Level of education, lifestyle, and morbidity in two groups of white collar workers. Journal of epidemiology and community health 1992; 46: 403-8.

6. Liu X, Bai H, Shi W, Shi J. Frozen-thawed embryo transfer is better than fresh embryo transfer in GnRH antagonist cycle in women with 3-10 oocytes retrieved: a retrospective cohort study. Archives of gynecology and obstetrics 2019; 300: 1791-6.

7. Liu X, Shi W, Shi J. Natural cycle frozen-thawed embryo transfer in young women with regular menstrual cycles increases the live-birth rates compared with hormone replacement treatment: a retrospective cohort study. Fertility and sterility 2020; 113: 811-7.

8. Oshio T, Kan M. Educational level as a predictor of the incidences of non-communicable diseases among middle-aged Japanese: a hazards-model analysis. BMC public health 2019; 19: 852. 
9. Baker DW, Gazmararian JA, Williams MV, Scott T, Parker RM, Green D et al. Functional health literacy and the risk of hospital admission among Medicare managed care enrollees. American journal of public health 2002; 92: 1278-83.

10. Scott TL, Gazmararian JA, Williams MV, Baker DW. Health literacy and preventive health care use among Medicare enrollees in a managed care organization. Medical care 2002; 40: 395-404.

11. Almeida NKO, Pedreira CE, Almeida R. Impact of maternal education level on risk of low Apgar score. Public health 2016; 140: 244-9.

12. Moreau C, Bouyer J, Ducot B, Spira A, Slama R. When do involuntarily infertile couples choose to seek medical help? Fertility and sterility 2010; 93: 737-44.

13. Wang J, Xue M. Influence of age, stigma and social support on male temporary ejaculation failure on IVF oocyte retrieval day. 2021; 19: 9.

14. Castro Martín T. Women's education and fertility: results from 26 Demographic and Health Surveys. Studies in family planning 1995; 26: 187-202.

15. Cohen JE, Kravdal Ø, Keilman N. Childbearing impeded education more than education impeded childbearing among Norwegian women. Proceedings of the National Academy of Sciences of the United States of America 2011; 108: 11830-5.

16. Everywoman J. Cassandra's prophecy: why we need to tell the women of the future about age-related fertility decline and 'delayed' childbearing. Reproductive biomedicine online 2013; 27: 4-10.

17. Sydsjö G, Selling KE, Nyström K, Oscarsson C, Kjellberg S. Knowledge of reproduction in teenagers and young adults in Sweden. The European journal of contraception \& reproductive health care : the official journal of the European Society of Contraception 2006; 11: 117-25.

18. Jin X, Wang G, Liu S, Zhang J, Zeng F, Qiu Y et al. Survey of the situation of infertile women seeking in vitro fertilization treatment in China. BioMed research international 2013; 2013: 179098.

19. Mahalingaiah S, Berry KF, Hornstein MD, Cramer DW, Missmer SA. Does a woman's educational attainment influence in vitro fertilization outcomes? Fertility and sterility 2011; 95: 2618-20.

\section{Hosted file}

table.pdf available at https://authorea.com/users/373747/articles/522398-impact-of-maternaleducation-level-on-live-birth-rate-after-in-vitro-fertilization-a-retrospective-cohortstudy 